\title{
Germanica
}

\section{Erinnerung und Zeitkritik bei Ingeborg Bachmann, Gert Hofmann und Botho Strauß}

Souvenir et critique du temps chez Ingeborg Bachmann, Gert Hofmann et Botho Strau $\beta$

Dirk Göttsche

\section{OpenEdition}

Journals

Édition électronique

URL : http://journals.openedition.org/germanica/2172

DOI : 10.4000/germanica. 2172

ISSN : 2107-0784

Éditeur

Université de Lille

Édition imprimée

Date de publication : 31 décembre 1993

Pagination : 79-95

ISSN : 0984-2632

Référence électronique

Dirk Göttsche, «Erinnerung und Zeitkritik bei Ingeborg Bachmann, Gert Hofmann und Botho Strauß », Germanica [Online], 13 | 1993, Online erschienen am: 07 Februar 2014, abgerufen am 06 Oktober 2020. URL : http://journals.openedition.org/germanica/2172 ; DOI : https://doi.org/10.4000/germanica.2172

Ce document a été généré automatiquement le 6 octobre 2020.

(c) Tous droits réservés 


\title{
Erinnerung und Zeitkritik bei Ingeborg Bachmann, Gert Hofmann und Botho Strauß
}

\author{
Souvenir et critique du temps chez Ingeborg Bachmann, Gert Hofmann et Botho \\ Strau $\beta$
}

Dirk Göttsche

1 Eine krisenartige Phase der Vertiefung des modernen Problemzusammenhangs von Bewußtseinsdarstellung, Zeitkritik und Erzählreflexion steht in den späten 60er Jahren am Übergang zu einer Gegenwartsliteratur, die sich «am Ende der Moderne» sieht und für die die Literaturgeschichtsschreibung Ingeborg Bachmanns Roman «Malina» bzw. jenes «Todesarten»-Projekt, aus dem dieser Roman als die «Ouvertüre» ${ }^{1}$ des geplanten, aber unvollendeten Zyklus hervorging, als wegweisend anzusehen beginnt ${ }^{2}$. Während zugleich ein Bezug zwischen den «drei großen NS-reflexiven Projekte[n] der deutschen Nachkriegsliteratur, Bachmanns "Todesarten", Johnsons "Jahrestage[n]" und Weiss' "Ästhetik des Widerstands"» und den «neuen Schreibweisen des Erinnerns» in den 80er Jahren hergestellt wird, für die Gert Hofmanns Erzählwerk steht ${ }^{3}$, scheint die von Botho Strauß repräsentierte gleichzeitige 'Postmoderne' die 'moderne' Engführung von Erinnerung, Subjektivität und Zeitkritik grundsätzlich in Frage zu stellen. Vor diesem Hintergrund analysiert der vorliegende Beitrag Romane und Erzählungen von Ingeborg Bachmann, Gert Hofmann und Botho Strauß, die in Anknüpfung an Traditionen der literarischen und philosophischen Moderne und im Rückgang auf Kriegs- und Nachkriegszeit Subjektproblematik, Zeitkritik und Selbstreflexion des Erzählens in je unterschiedlichen ästhetischen Ausfaltungen einer Dialektik der Erinnerung vermitteln. 


\section{Die Geschichte im Ich: Ingeborg Bachmanns Roman «Malina»}

2 Bereits in ihren «Frankfurter Vorlesungen» (1959/1960) schreibt Ingeborg Bachmann dem modernen Subjekt die Erfahrung zu, «daß es sich nicht mehr in der Geschichte aufhält, sondern daß sich neuerdings die Geschichte im Ich aufhält» (IV, 230) ${ }^{4}$. Die Erfahrung der unauflöslichen Durchdringung von Subjekt und Zeitgeschichte wird in der Erzählung «Jugend in einer österreichischen Stadt» (1959) in einem an Walter Benjamin erinnernden poetischen Moment «bewegungslosen Erinnern [s]» (II, 93) gestaltet, der im Augenblick des Abschieds Kindheitserinnerung und österreichische (Kriegs-) Geschichte zusammensieht, während die Verrückte' Durchdringung von Geschiehtsreflexion, Zeitgeschichte und Krankheitsdarstellung in dem Prosatext der Büchnerpreisrede «Ein Ort für Zufälle» (1964) eine solche bildhafte Verdichtung nicht mehr kennt. Der genetisch wie motivisch bereits im Projektzusammenhang der «Todesarten» stehende Berlin-Text ${ }^{5}$ zeigt die Vertiefung der Zeitkritik zu einer grundlegenden Auseinandersetzung mit der «Geschichte im Ich» und den damit einhergehenden Konzeptionswandel von Ingeborg Bachmanns Schreibweise, der in «Malina» (1971) jenes komplexe reflexive, dialektische und musikalische Erzählverfahren hervorbringt, das über den modernen Realismus der Nachkriegsliteratur hinausführt. Die Entwicklung von Ingeborg Bachmanns Prosawerk - und d.h. insbesondere auch der Weg von dem Romanprojekt der 50er Jahre um den Kriegsheimkehrer Eugen Tobai über den ersten, als ein österreichischer Zeitroman einsetzenden «Todesarten» - Roman um einen gleichnamigen Historiker und Psychologen $(1963 / 65)^{6}$, über die allegorische Verknüpfung von Wüstenreise und Krankheit, Zerstörung des anderen und nationalsozialistischen Vernichtungspraktiken in dem Romanfragment um Franziska Ranner (1965/66) zu der Dekonstruktion herkömmlicher Erzählverfahren in dem Bewußtseins- und Zeitroman «Malina» spiegelt als ein immer wieder neu ansetzender literarischer Arbeitsprozeß mit besonderer Eindringlichkeit die Vertiefung der Erinnerungskonzeption in der Geschichte des Nachkriegsromans, indem die Erfahrung von Krieg und Nationalsozialismus zum Paradigma von Gewalterfahrung schlechthin wird. Dieser Intensivierungsprozeß reicht bis in die Arbeit an der endgültigen ästhetischen Gestalt des «Malina»-Romans. Vor der Drucklegung vorgenommene Kürzungen treffen insbesondere drei Textteile, die ursprünglich das Kapitel «Von letzten Dingen» im Stil eines herkömmlichen Zeitromans einleiten sollten: Eine satirische Stadtrundfahrt, die unter dem Titel «Besichtigung einer alten Stadt» gesondert veröffentlicht wurde ${ }^{7}$, ein Besuch ausländischer Gäste, die sich nach «flower power» in Wien erkundigen, und der Fall eines Gymnasiasten, der Opfer eines nach nationalsozialistischen Riten inszenierten kollektiven Selbstmordversuchs dreier schüler wird und dadurch drastisch das Fortwirken der öffentlich verdrängten nationalsozialistischen Vergangenheit allegorisiert ${ }^{8}$.

3 Die Vertiefung der Problemstellung und die Verdichtung der ästhetischen 'Komposition' lassen den Roman jedoch gerade nicht zu der «Geschichte einer schönen Seele» ${ }^{9}$ werden, wie die in den Schemata von Gesellschaftskritik vs. Neue Subjektivität befangene Literaturkritik seinerzeit glaubte. Das mittels eines Zitats aus Hölderlins «Fragment von Hyperion» formulierte Leitmotiv des Ringens um die «verschwiegen[e] Erinnerung» (III, 23) und ihre Erzählbarkeit - «Ich muß erzählen. Ich werde erzählen. 
Es gibt nichts mehr, was mich in meiner Erinnerung stört» (III, 23 u.ö.) und gegenläufig «Ich will nicht erzählen, es stört mich alles in meiner Erinnerung» (III, 27 u.ö.) - zielt nicht auf die anekdotischen Erinnerungen eines sich seiner Geschichte und seiner geschichtlichen Erfahrung sicheren Subjekts, sondern vielmehr auf die schwierige Annäherung an den verborgenen 'Grund' eines exemplarischen Ich, das sich um so deutlicher als abgründig erfährt, je tiefer es sich erinnernd zu begründen sucht, indem es in seiner Geschichte die "Geschichte im Ich» erkennt. Die in dem Franza-Roman zunächst noch getrennt gestellten Fragen nach der Geschichte der erlittenen Lebenszerstörung («Wann hat es angefangen ?» III, 401) und nach dem Zusammenhang von Lebens- und Zeitgeschichte («Meine Geschichte und die Geschichten aller, die doch die große Geschichte ausmachen, wo kommen die mit der großen zusammen» III, 433) bilden in dem komplexen Reflexionsraum des «Malina» - Romans zwei Seiten einer Problemstellung ${ }^{10}$.

4 Erinnerung steht damit in «Malina» grundsätzlich in der Ambivalenz von Selbstbegründung und Selbstgefährdung, das Erzählen von Erinnerung dokumentiert als der Versuch des Ich, sich eine Geschichte zuzuschreiben und darin zugleich eine Zukunft zu entwerfen, die Geschichte der Vernichtung des Ich. Die Schuldfrage, die der ursprüngliche Umschlagtext bewußt mit der falschen Alternative «Mord oder Selbstmord ?» stellt, bleibt offen, da der Schlußsatz «Es war Mord» (III, 337) zwar offenbar nicht mehr von dem vorgeführten Ich gesprochen wird, sondern von einem abstrakten Erzähler-Tch', das bereits in dem einleitenden Personenverzeichnis das sich erinnernde und reflektierende Ich setzt, dieses abstrakte 'Ich' der erzählerischen Konstruktion jedoch weder Konturen gewinnt noch auktorial über den 'Sinn' des dargestellten 'Untergangs' (III, 240) verfügt. Dessen scheinbar abschließende Bewertung als «Mord» führt vielmehr in den vorgeführten Erinnerungs- und Reflexionsprozeß zurück, der sich in drei 'Akten' je unterschiedlicher Formen der Erinnerung und der Zeit- und Bewußtseinskritik entfaltet.

5 Nach der Exposition der "Gedankenbühne» (III, 286) in dem Vorkapitel (der Konstellation Ich / Malina als Doppelgängerfiguration, des imaginären Raumes des «Ungargassenlandes» und der Kunst-Zeit des «heute») erzählt das Kapitel «Glücklich mit Ivan» einen mit Kierkegaards Begriff der Wiederholung beschreibbaren Versuch der «Erlösung» des Lebens in der Utopie eines «ganzefn] Leben[s]» der Liebe (III, 48f.). Das «Pathos» dieser Projektion (III, 49) erweist sich jedoch von Anfang an als pathologisch, die Liebe als «Injektion» (III, 45), die 'Wiederholung' als lebendige Vergegenwärtigung vorgängiger Verletzungen, die das Ich schon mitten im «Ivanleben» des «heute» auf die Geschichte seiner Zerstörung zurückverweisen. Der 'kritische' Zustand des Ich wird in der Österreichkritik des MühlbauerInterviews zeitkritisch gewendet, wenn das Ich seine Zeit durch den «täglichefn] Brand des Justizpalastes» (III, 90) gezeichnet sieht und der gewalterfüllten Gegenwart die Utopie eines Gegensätze und Grenzen überbrückenden «Haus [es] Österreich» entgegenstellt (III, 99). Daß dieser Rückblick auf das alte Kaiserreich als Projektion zu verstehen ist, zeigt nicht zuletzt die Wolfgangsee-Episode mit ihrer literarisch gesättigten Satire der österreichischen 'high society' und ihrer leerlaufenden «Konversation» (III, 152-170).

6 Die Lektüre von Nietzsches Schrift «Ecce Homo. Wie man wird, was man ist» (III, 170) und des Katalogs des Heeresgeschichtlichen Museums, in dem Malina arbeitet (III, 171), bereiten am Ende des Ivan-Kapitels die Rückwendung des Ich auf seine eigene Geschichte und Geschichtlichkeit und deren Reflexion in den Dialogen mit Malina vor. 
Daß die Erinnerung traumatischer Erfahrung im Medium der Traumsprache und in dem Ringen mit einer «Vater»-Gestalt, die «nicht mein Vater ist» (III, 179), nicht psychoanalytisch aufgelöst werden darf, sondern gerade in ihrer radikalen Subjektivität auf die literarische Darstellung repräsentativer Muster struktureller Gewalt zielt, verdeutlicht schon die einleitende Setzung, die das Traumkapitel aus der trotz allem noch in die historische Realität der Gegenwart eingelassenen Kunstzeit des «heute» heraushebt: «Es ist ein Ort, der heißt Überall und Nirgends. Die Zeit ist nicht heute. [...] Für die Einheiten dieser Zeit, in die andere Zeiten einspringen, gibt es kein Maß [...]» (III, 174). Während Franziska Ranners traumatische Erinnerung ihrer «jordanischen Zeit» noch auf die Entlarvung Jordans als der Personifizierung eines Verhaltens zielte, das begrifflich als «Faschismus» gefaßt wurde (III, 403), zitieren die einzelnen Vernichtungsträume des Ich in der Form von Vergasung, Gefangenschaft, Deportation, Mißhandlung etc. zwar Erfahrungen, die unauflöslich mit der Erinnerung katastrophischer Geschichtserfahrung und insbesondere der Shoah verbunden sind, stellen sie jedoch neben andere physische und psychische Gewalterfahrungen und führen scheinbar unausweichlich zu Malinas universaler Sicht der Geschichte wie der «Geschichte im Ich» als 'Kriegsgeschichte': «Es ist Krieg. Und du bist der Krieg. Du selber» (III, 185) und «Es ist der ewige Krieg» (III, 236). Die Einsicht, «hier wird man ermordet» (III, 236), meint dann aber nicht mehr einen personalen «Mörder», sondern einen Abgrund der Gewalt in der scheinbar gesicherten Ordnung der modernen Gesellschaft, der in den Verbrechen des Nationalsozialismus mit katastrophischer Macht hervorgetreten ist, alltäglich jedoch in den sozialen Bezügen eines jeden aufzubrechen vermag. In Anlehnung an J.A. Barbey d'Aurevilly hatte Ingeborg Bachmann diese latente Permanenz der Gewalt in der zivilisierten Gesellschaft in ihren Entwürfen zu einer Vorrede zum Franza-Roman als die sublimen Verbrechen des Geistes bezeichnet, um deren Darstellung es ihr in den «Todesarten» gehe (III, 342) ${ }^{11}$. Das im wörtlichen Sinne radikale Erinnerungsverfahren des Traumkapitels wirft die Frage auf, wie der einzelne und die Gesellschaft mit der freigelegten Abgründigkeit ihrer Selbstentwürfe und Ordnungen umgehen bzw. «überleben» (III, 223). Dem Kapitel «Von letzten Dingen» kommt daher die Aufgabe zu, die apodiktische Universalität der Traumdeutung wieder mit dem vergelichen Glücksentwurf des Ich und seiner Lebensgeschichte in ihrem historischen und gesellschaftlichen Raum zu vermitteln. Diese Reflexion erfolgt im Dialog der in der Konstellation Ich/Malina entfalteten Dialektik gegensätzlicher und zugleich aufeinander verwiesener Sprech- und Verhaltensformen, und zwar anhand von Buch- und Zeitungslektüren, in der Erinnerung an die Nachkriegszeit als «Mordschauplatz» (III, 259) und «Reigen» einer «universeile [n] Prostitution» (III, 274f.) und im Nachdenken über die wachsende Entfremdung zwischen Ich und Ivan, die das Ich endgültig aus seinem «Ivanleben» auf sein «Malinafeld» (III, 284) der Selbstreflexion und des Schreibens verweist.

7 Schon im Traumkapitel besteht trotz der Zustimmung des Ich zu der Kriegsmetaphorik Malinas ein notwendiger Gegensatz zwischen Malinas auf Fakten und Feststellungen drängendem Fragen und dem bildhaften Erinnern des Ich: «man kann doch nur erzählen, was man sieht» (III, 236). Die Gegensätzlichkeit der Malina und dem Ich zugeordneten Erinnerungs- und Erzählkonzepte verschärft sich nicht zufällig an jenem Punkt der Dialoge «Von letzten Dingen» zu einer prinzipiellen Konkurrenz, an dem es (autoreflexiv) um die literarische Darstellung der Vernichtungserfahrungen als «Todesarten» geht (III, 288-290). Die hier einsetzende Markierung der Dialogbeiträge des Ich mit expressiven musikalischen Vortragsbezeichnungen, der durchgängige 
musikalische Anspielungshorizont des Romans und das immer wieder neu ansetzende, sich an Lektüren, Gegenständen und Erinnerungen entzündende und so zu keinem Abschluß kommende Erzählen des Ich stehen für eine imaginative Form des Umgangs mit der «verschwiegenen Erinnerung» und mit der universalen Gewalterkenntnis des Traumkapitels, die sich jedoch beständig gegenüber der Herausforderung ihres dialektischen Gegenteils, des denkenden Geistes (siehe III, 304 und 334), «klären» (III, 23) muß, bevor das Ich am Ende die Kraft zu dieser Entgegensetzung verliert und mit einem Zitat aus Rimbauds «Une saison en enfer» resigniert: «Nous allons à l'Esprit !» (III 334) Demgegenüber verkörpert die Malina-Figur des «Malina» - Romans als Historiker im Heeresgeschichtlichen Museum und als das alter ego des imaginativen Ich einen in besonderer Weise «verschwiegenen» Umgang mit der «Todesarten»Thematik, da er «nie ein Wort aus seinem Leben sagen» würde (III, 299), sich auf die Erkenntnis und Archivierung der Gewalterfahrung beschränkt und sie allenfalls in 'apokrypher' Form (III, 11) veröffentlicht. Diese Entgegensetzung wird mit den Motiven des Dimorphen (III, 278), der Zerrissenheit zwischen Männlichem und Weiblichem, Verstand und Gefühl (III, 248), mit der Dialektik von Schönheit und Geist (III, 277, 304), Licht und Dunkel (III, 289), Ruhe und Unruhe (III, 310), Siegen und Ergriffensein (III, 311/313) etc. immer neu umspielt, ohne jedoch begrifflich vereindeutigt zu werden und ohne daß diese Bewegung dialektischer Entgegensetzung zum Stillstand käme. Die Asymmetrie, daß das Ich auf sein dialektisches Gegenteil Malina angewiesen scheint, Malina jedoch nicht seinerseits auf das Ich, wird auf der Ebene der Romankomposition dadurch aufgehoben, daß die «klare» Stimme Malinas ohne die «dunkle Geschichte» des Ich (III, 22f.) nicht zu hören wäre: Erst in der Gegenbewegung gegen das imaginative Sprechen des Ich in seinem Ringen um die Erzählung der «verschwiegenen Erinnerung» und in seinem lebendigen Aufbegehren gegen die Universalität der Gewalt findet Malinas Zuwendung und Abgrenzung ihre Berechtigung. Trotz des Untergangs des Ich hebt der Romanschluß diese Dialektik der in dem Ich und Malina gestalteten Stimmen, Erinnerungs- und Erzählkonzepte nicht auf.

\section{Lebensgeschichte und Zeitkritik bei Gert Hofmann}

8 Die literarische Kritik an der Geschichtsvergessenheit der bundesdeutschen Gesellschaft und ihrer «institutionalisierten "Vergangenheitsbewältigung"» ${ }^{12}$ durch die Aufdeckung verdeckter Gewaltstrukturen am Paradigma des Nationalsozialismus steht im Mittelpunkt auch von Gert Hofmanns Erzählprosa. Vom Hörspiel kommend, arbeitet Hofmann mit unterschiedlichen Formen eines dialogischen Erzählens, das wie jenes des «Malina» - Romans durch die Auflösung der Erzählerposition, durch eine durchgängige autoreflexive Brechung der Darstellung und durch die Gegeneinanderführung dialektisch aufeinander bezogener Stimmen gekennzeichnet ist. In dem Roman «Unsere Eroberung» (1984) und der Erzählung «Veilchenfeld» (1986) bleibt der zeitkritische Gegenwartsbezug elliptisch und wird nur durch die sprach- und bewußtseinskritische Form der erzählerischen Inszenierung des kindlichen Zeugenblicks wirksam, durch den diese Texte geschichtliche Erfahrung als lebensgeschichtliche Prägung perspektivieren. Die Novelle «Die Denunziation» (1979) und der Roman «Unsere Vergeßlichkeit» (1987) vermitteln dagegen Geschichtsreflexion und Zeitkritik in der Selbstreflexion schreibender Subjekte, deren 
Lebensentwürfe durch die Konfrontation mit der Erinnerung von Lebens- und Zeitgeschichte grundlegend in Frage gestellt werden ${ }^{13}$.

In der Novelle «Die Denunziation» sieht sich der - von einer wie in «Malina» konturenlosen Vermittlerinstanz vorgeführte, im Rahmen einer Brieffiktion über sich reflektierende - Rechtsanwalt Karl Hecht als ein Repräsentant der in verantwortliche Positionen aufgerückten Generation der 'Söhne' nach dem Tod seines in einer psychiatrischen Anstalt in New York verstorbenen Zwillingsbruders Wilhelm durch dessen Aufzeichnungen, literarische Entwürfe und historische Recherchen auf seine vergessene bzw. verdrängte Kindheit in den späten Kriegsjahren verwiesen, zumal ihm die erzählerische Konstruktion der Novelle kein Ausweichen vor der sich aufdrängenden Erinnerung ermöglicht, da sowohl der Fall, mit dem der Anwalt sich in der dargestellten Nacht der Selbstreflexion zu befassen hat, als auch der Konflikt mit seinem ältesten Sohn, der ihm den Rückzug in einen familiären Privatbereich verwehrt, die von dem Zwillingsbruder aufgezeichneten Gewalterfahrungen spiegeln. Die Zerstörung der elterlichen Familie durch den Nationalsozialismus infolge einer Denunziation im Herbst 1944, der juristische Fall um einen linksorientierten Lehrer in der ideologisierten Atmosphäre des Radikalenerlasses von 1972 und der Konflikt mit dem «radikalpolitische[n] Sohn», der dem Vater zufolge «mit einem aufgeklappten ideologischen Rasiermesser in der Tasche herumgeht» $(\mathrm{D} 72,85)^{14}$, erweisen sich als die «drei schweren Fälle», zwischen denen der Anwalt «ständig hin- und hergehetzt» wird (D 77). Die Novelle gestaltet diese ständige Vermittlung von Kindheitserinnerung, beruflicher und privater Gegenwart, von Lebensgeschichte und Zeitgeschichte durch das Eingangsmotiv einer versehentlichen Vermischung der juristischen Fallunterlagen mit den Aufzeichnungen des Bruders (D 8), so daß die Hin- und Herbewegung zwischen den sich gegenseitig spiegelnden Fällen $\mathrm{zu}$ dem Versuch wird, «alles wieder auseinanderzuklauben» (D 10), um jene Infragestellung des bürgerlichen Selbst- und GeschichtsVerständnisses abzuwehren, welche die drei Gegenfiguren, die alle den Namen Wilhelm tragen, repräsentieren.

10 Eine solche Ausgrenzung verhindert wie in «Malina» aber auch hier die Dialektik der Erinnerung, da Hechts Ordnungsbemühen auf die Lektüre des zu Ordnenden angewiesen ist und daher immer tiefer in jene Verunsicherung hineingerät, gegen die sich seine Hin- und Herbewegung ohnmächtig wehrt. Die Aufzeichnungen des in die Fremde der Erinnerung ausgezogenen Bruders, seine Briefentwürfe mit dem vergeblichen Bemühen, die Vorgänge um die Denunziation des Vaters durch die Befragung von Zeugen und möglichen Schuldigen nachträglich aufzuklären, und die Verrückten' «Zettel», die das Scheitern seiner erinnernden Selbstbegründungsanstrengung dokumentieren, brechen durch jenes «unergründlich[e] Loch» ein, jene «schreckliche Finsternis» des Vergessens, die der zu Hause gebliebene Hecht «hinter» sich spürt (D 14). Die Erinnerungen des Zwillingsbruders stellen als «meine Erinnerungen [...], wenn auch sozusagen von der anderen Seite her» (D 37) die andere Stimme der Novelle dar, die bis zu der Verbrennung der Aufzeichnungen (D 72) mit Mitteilungen aus dem 'Fall Treterle' wechselt, aber auch in dem zwischen dem 'Fall' und der Vater / Sohn-Problematik hinund hergehenden Schlußteil präsent bleibt: durch jene «Zettel» (D 74, 77f., 79, 85), die das Zerbrechen Wilhelms an der Ausweglosigkeit eines Erinnerns zeigen, das von der Vergangenheit nicht 'loskommt' (D 17) und sich angesichts der 'Vergeßlichkeit' der Zeitzeugen und des öffentlichen Bewußtseins schließlich selbst verrückt glaubt. Das 
Motiv der «langerwarteten Untersuchung meines Kopfes» (D 85), das der Roman «Unsere Vergeßlichkeit» als Chiffre für die Selbstaufgabe des Bewußtseins wieder aufgreifen wird (UV 295-301), gestaltet die Deformation des Erinnernden durch die Verdrängungsleistung eines öffentlichen Bewußtseins, das konkretes Geschichtsbewußtsein als 'abweichende Ansicht' (D 79) denunziert. Hechts Einsicht in die Fragilität verantworteten Lebens - «das Vergängliche, gestützt auf einen Hauch» (D 89) - zielt als Feststellung, «wie hauchdünn die Schicht der guten Manieren und der Vernunft bei uns in Wahrheit ist» (D 84), nicht auf einen reflektierten Umgang mit dem Abgrund der Gewalt in der sozialen Ordnung oder auf die Anerkennung des anderen, sondern auf einen aufgeklärten Zynismus, der die moralische Frage verantwortlichen Handelns suspendiert: «Denn ohne Zweifel [...] leben wir wie eh und je in einer von unübersehbaren Kräften beherrschten und dunklen Gesetzen unterworfenen Welt [...]» (D 82). Gegen die zynische Resignation setzt sich die von Wilhelm verkörperte 'andere Seite' der Erinnerung jedoch als 'Störung' (D 93) erneut durch, indem das Motiv des unterirdischen Flusses und seines warnenden Rauschens, an das Wilhelm sich aus der Kinderzeit erinnert (D 12f.), seinem Bruder Karl, der erkennt, daß auch er die «unterirdischen Verästelungen [...] die ganze Zeit in [sich] getragen haben muß» (D 91), in der apokalyptischen Vision einer Sintflut überfällt. Die Anstrengung der Verdrängung bleibt auf die in mythischen Bildern wiederkehrende Erinnerung verwiesen.

11 Der Roman «Unsere Vergeßlichkeit» führt die Kritik mangelnden Geschichtsbewußtseins einerseits fort - «Wir, mit unseren Erfahrungen, sind nicht mehr gefragt [...]» (UV 287) -, bettet sie jedoch andererseits in einer grundsätzlicheren und trotz konkreter Schreckensbilder aus den letzten Kriegstagen abstrakteren Reflexion des Zusammenhangs von Erinnerung, Identität und Geschichte ein, indem am Leitfaden eines autoreflexiven Motivs - ein Schriftsteller-Ich ist mit seinem Roman «Unsere Vergeßlichkeit» unterwegs zu seinem Lektor, dem jedoch weniger an dem Roman selbst als an publizierbaren Exempeln seiner klischeehaften Literaturtheorie einer literarischen Erweiterung «reduzierte [r] Wahrnehmung» (UV 22) gelegen ist zwei Gestalten der Dialektik von Erinnern und Vergessen gegeneinander geführt werden, die sich als gegensätzliche und doch komplementäre Reduktionsformen der Erinnerung erweisen. Die Ich-Gestalt, die ihre «Heilpraktikerexistenz [...] in eine Schriftstellerexistenz auf[zu]heben» trachtet (UV 37) und sich in ihrem Roman-imRoman therapeutisch «selbst als Beispiel» nimmt (UV 168), arbeitet sich an dem «Verschwinden von Gelebtem» in der «Finsternis, in die gelebte Augenblicke und damit wir selber immerzu zu versinken drohen und dann tatsächlich versinken» ab (UV 62). Sie führt darin den vergeblichen Versuch vor, dem bei deralltäglichen Verrichtung des Staubwischens in grotesker Grundsätzlichkeit aufgebrochenen «Vergessensloch» (UV 86) durch ein «Leben von völliger Bewußtheit und fortwährender Durchdringung» (UV 172) entgegenzuwirken. Dieser Versuch vollkommener Gegenwärtigkeit, in dem der Schriftsteller sich gegen die von dem Lektor geforderte Proklamation einer neuen Wirklichkeit (siehe UV 54) als «rückwärts gewendet» (UV 49) erfährt, stellt sich nicht nur als «eine todsichere Methode, verrückt zu werden» (UV 175) dar, da der gelebte Augenblick dem Versuch seiner vollkommenen Vergegenwärtigung immer schon «rasend» entläuft (siehe UV 94), sondern auch als eine gewaltsame Vereinnahmung anderer (der Frau und des Sohnes), deren Zeugenschaft der Schriftsteller in seinem eifersüchtigen Kampf gegen die naturgegebene 'Vergeßlichkeit' des Lebens erzwingt, 
bis er vor der notwendigen Unvollständigkeit und Individualität aller Erinnerung kapituliert (UV 205, 233).

Der «Hang zur Unendlichkeit» (UV 169), der sich in seinem unabschließbaren Kampf gegen die «Vergeßlichkeit» ausdrückt, spiegelt sich in den vergeblichen Versuchen, einen Roman zu rechtfertigen, in den er alle «Wirklichkeiten» (UV 61), die sich ihm aufdrängen - und unverzüglich auch Reißer (UV 66), seinen Führer durch die 'Unterwelt' der Geschichte - aufnehmen zu müssen glaubt, da er am liebsten «Über alles schreiben» würde, «weil jedes zum ganzen gehört» (UV 168). Dieser grotesken Hypertrophie der 'ganzen' Erinnerung und des vollkommenen Buchs - verzweifelter Ausdruck der 'Leere' hinter dem Roman-im-Roman (UV 28) und der tödlichen «Stille» (UV 227) der ganz in seiner «Misere» (UV 47) verrannten Schriftstellerexistenz - steht in Reißer die nicht minder groteske Verkörperung einer verstörenden Zeugenschaft der Trümmer und Opfer der letzten Kriegstage gegenüber, von denen die geschiehtsvergessene Wiederaufbaugesellschaft nichts mehr wissen will, auf deren Boden sie sich jedoch architektonisch wie geschichtlich erhebt. Diese historische Zeugenschaft ist als Deformationsform der «Geschichte im Ich» gestaltet, als Hybris obsessiver Erinnerung, die in ihrem verzweifelten Kampf gegen das «Schweigen» (UV 285), mit dem die Nachkriegszeit «über alles hinweg[geht]» (UV 185), die Gegenwart verliert (siehe UV 253). Reißer, der in der «oberen» Stadt der Gegenwart nur die «untere», «die alten Bilder» (UV 185) zu sehen vermag und seinen «Kopf voller Toter» hat (UV 254), wird, von den «Stimmen» der Opfer (UV 215 u.ö.) getrieben, zur lebendigen Allegorie des vergeblichen Versuchs, die Vergangenheit vollständig 'heraufzuholen' (UV 287) und die Frage zu beantworten, «wie alles gekommen ist» (UV 211). Für die das Historische ergänzende moralische Dimension dieser Zeitkritik steht wie in der «Denunziation», deren Konstellation der Roman «Unsere Vergeßlichkeit» in zugespitzter Form variiert, und zugleich an Ingeborg Bachmanns «Todesarten»-Projekt erinnernd - die Gestalt des Sohnes des Romans-im-Roman, der in dem Verhalten seiner Mitschüler gegenüber einem psychiatrisch betreuten Lehrer den «reinste[n] Faschismus» entdeckt (UV 243).

13 Wenn die einleitende Behauptung des Schrifstellers, ihm sei es endlich «gelungen, [seinen] Roman UNSERE VERGESSLICHKEIT abzuschließen» (UV 7), im Zuge des dargestellten Stadtgangs in immer neuen Rechtfertigungsversuchen und Neufassungsideen in die Vorführung seiner Unabschließbarkeit überführt wird, so ist Reißer im Umgang mit seiner obsessiven Erinnerung auf die unendliche Wiederholung seiner Gedächtnissgänge und -erzählungen festgelegt, ohne sich dadurch je von dem Schrecken der Erinnerung befreien zu können. Wie sehr die in der Ichgestalt und in Reißer in grotesker Überzeichnung gegeneinander gesetzten Deformationsformen der Erinnerung aufeinander bezogen sind, zeigt nicht nur die deutliche Doppelgängerschaft der Figuren (siehe UV 167), sondern auch das Wechselspiel ihrer Monologe - der Schriftsteller erzählt Reißer seinen Roman, dieser führt jenen immer tiefer «in die Tiefe» (UV 249) der überbauten Trümmerwelt der Vergangenheit -, mit denen sie sich jeweils an neuralgischen Punkten verborgener Bezüge ins Wort fallen, ohne je zu einem Dialog im eigentlichen Sinne zu gelangen. Die Gegeneinanderführung ihrer Stimmen, die Hofmann zunächst in dem Hörspiel «Die Überflutung» (1979) bzw. in den Erzählungen «Fuhlrotts Vergeßlichkeit» und «Unsere Vergeßlichkeit» (1981) getrennt entworfen hatte ${ }^{15}$, endet mit der Rückkehr Reißers in das «Labyrinth» (UV 116) seiner Gedächtnisgänge und mit dem Sich-Verlaufen des Schriftstellers in dem Wahn der Möglichkeit einer objektiven, medizinisch-physiologischen 'Begründung' der 
Erinnerung in der «Tiefe» des Gehirns (UV 298f.), in der für die moralische «Tiefe» der Lebens- und Zeitgeschichte kein Raum mehr bleibt. Zwarbesitzen die Figuren selbst ein Bewußtsein ihres dialektischen Verhältnisses: Wie der Schriftsteller sich durch das Hin- und Herbewegen zwischen seinem Roman und den Mitteilungen Reißersgrundsätzlich verunsichert sieht («[...] wenn Reißer schreibenwürde, was er uns erzählen könnte !» UV 218), so kontrastiert Reißer seine Erinnerung des «öffentliche[n] Unglück[s]» mit dem«privat[en] Malheur» des Schriftstellers (UV 289) - doch reichen die Ansätze zu einer Öffnung der monologischen 'Erzählungen' nicht für Annäherung und Ausgleich; die Zusammenführung der in den Stimmen Ich und Reißer aporetisch gefaßten Zerrformen der Erinnerung erfolgt allein durch die Komposition des Romans.

\section{Zeit-Reflexion in Botho Strauß' Roman «Der junge Mann»}

Der in den Werken Ingeborg Bachmanns und Gert Hofmanns aufgezeigten strukturellen Dialektik gegensätzlicher Formen des Erinnerns und Erzählens steht in dem Roman «Der junge Mann» (1984) von Botho Strauß eine Konstellation aus relativ selbständigen und doch motivisch eng miteinander verknüpften Kapiteln und Welten gegenüber, die der 'postmodernen' Erzählerdeutung der «Einleitung» zufolge «dem Diversen seine Zonen schaffen» sollen (JM 10) ${ }^{16}$ und sich als Variationen und Gestaltungsformen des Themas der Gleichzeitigkeit erweisen ${ }^{17}$. Die einzelnen Kapitel wie die in sie eingelegten «Ideen-Geschichten» (JM 123) führen die Figuren jeweils aus der 'linearen' Zeitform gesellschaftlicher Handlungsusammenhänge heraus auf die 'andere Seite' (JM 74) einer der «Einleitung» nach ursprünglicheren «Gleiche[n] Zeit» (JM 14): (1) in das «mehrzeitige Gefüge» des Theaters und sein «Zwielicht von einst und jetzt» (JM 51) ; (2) in einen allegorischen «Turm der Deutschen» voll der Stimmen aller Zeiten (JM 77-80) und in die Erfahrung der Wiedergeburt (JM 98); (3) in die phantastische Welt eines «Reservats» von Geschichts- und «Gesellschaftslosen» (JM 109); (4) in die Geschichtsallegorie einer «Terrasse», auf der sieben Figuren angesichts der «gewaltigen, unübersehbaren Zeit-Massen» der Geschichte sich erinnernd und über Erinnerung reflektierend dem «neuen Zeit-Prinzip» (JM 203) eines komplexen «ZeitRaums» (JM 235) als «Besinnung auf unseren Lebensgrund» (JM 212) zu nähern suchen, bevor sie sich in dem «Geistergarten» der «stillstehenden Zeit» (JM 308f.) verlaufen; und (5) in einen von der «Zeit-Lupe» des Augenblicks (JM 341) bestimmten «Zeit-Raum» «grenzenlose [n] Warten[s]» (JM 353). Der Bogen von dem euphorischen Entwurf der «Einleitung», gegen das «Regime des totalen öffentlichen Bewußtseins» (JM 10) und den «allesdurchbohrenden Zeit-Pfeil» (JM 14) in Anlehnung an den Zeitbegriff der modernen Physik «Schaltkreise [...] zwischen dem Einst und Jetzt» zu schließen (JM 11) und «die kostbaren Kristalle des Stillstands» zu retten (JM 15), zu dem unaufgelösten Nebeneinander der von Ossia und Leon Pracht vertretenen Kunstauffassungen (episodische 'Augenblicke' vs. geformte Geschichte) zeigt einerseits, wie der Roman seine Zeitkritik in einer grundlegenden Reflexion von Zeit und Zeitempfinden fundiert, andererseits jedoch, daß diese Zeit-Reflexion nicht in der 'postmodernen' Erzählerdeutung der «Einleitung» aufgeht. Die Fragwürdigkeit des scheinbar wieder ganz auktorialen Erzählers zeigt sich auch in seinem Begriff «RomantischerReflexionsRoman» (JM 15), der eine Wiederaufnahme des frühromantischen Projekts einer «progressiven Universalpoesie» suggeriert, während 
doch die Struktur des Nebeneinander und die Thematik der Gleichzeitigkeit der teleologischen und dialektischen Grundfigur der Poetik von Novalis und Friedrich Schlegel deutlich entgegengesetzt sind. Die Anspielungen auf Goethes «Lehrjahre» in der leitenden Leon Pracht-Handlung einer über den 'Umweg' des Theaters führenden 'Bildungsgeschichte' weisen vielmehr auf das Strukturmodell des Romans «Wilhelm Meisters Wander jähre oder Die Entsagenden», in dem Goethe die Komposition eines zeitkritischen Romans in einer Konstellation von unabhängigen und doch durch wechselseitige Spiegelungen, die intermittierende 'Rahmenhandlung' einer Entwicklungsgeschichte und einen Erzähler / Redaktor von eingeschränkter Autorität aufs engste aufeinander bezogenen Welten vorgeführt hat ${ }^{18}$. Hier knüpft Botho Strauß' «Junger Mann» als ein Roman über den Zeitgeist der Jahre 1969 bis 1984 (siehe JM 37, 347) und als 'ZeitRoman' über jenes «Element» «Zeit Zeit Zeit», mit dem «die Menschen immer noch am wenigsten zurecht [kommen]» (JM 7), an, indem er seine allegorischen und phantastischen Variationen der Zeitaufhebung in die Entwicklungsgeschichte von Figuren einläßt, die aus der «Wildnis zur Gleichen Zeit» (JM 96) - nicht zufällig durch den lebendigen Bezug zu anderen (siehe Leon/Yossica) - wieder herausfinden und so in Übertritt und Rückkehr eine narrative Ausfaltung der Dialektik von 'linearer' und 'gleicher' Zeit darstellen. Es ist damit angedeutet, daß die Kritik des öffentlichen Zeitund Geschichtsbewußtseins in dem Roman von Botho Strauß eine andere, im einzelnen durchaus problematische Vermittlung und Radikalisierung des 'modernen' Zusammenhangs von Subjektproblematik und Zeitkritik in einem durchgängig selbstreflexiven Erzählen darstellt, das die leitende Erinnerungsthematik Bachmanns und Hofmanns in einer übergreifenden Zeitthematik aufhebt.

\section{NOTES}

1. - Ingeborg Bachmann, Wir müssen wahre Sätze finden, Gespräche und Interviews. Hrsg. von Christine Koschel und Inge von Weidenbaum. München 1982, S. 95.

2. - Siehe Rainer Nägele: Geschichten und Geschichte. Reflexionen zum westdeutschen Roman seit 1965. In: Deutsche Gegenwartsliteratur. Ausgangspositionen und aktuelle Entwicklungen. Hrsg. von Manfred Durzak. Stuttgart 1981, S. 234-251, hier: S. 240; Klaus Briegleb und Sigrid Weigel: Einleitung. In: Dies. (Hg.): Gegenwartsliteratur seit 1968 (Hanser Sozialgeschichte der deutschen Literatur, Bd. 12), München 1992, S. $12 f$.

3. - Klaus Briegleb: «Aufbruch 1968». Der Mythos vom Neuanfang. In: Gegenwartsliteratur seit 1968 (Anm. 2), S. 19-150, hier: S. 106 bzw. 133.

4. - Ingeborg Bachmann: Werke. Hrsg. von Christine Koschel, Inge von Weidenbaum und Clemens Münster. München 1978, Bd. IV, S. 130. Aus dieser Ausgabe wirdunter Angabe des Bandes und der Seitenzahl zitiert.

5. - Vgl. Verf.: «Malina» und die nachgelassenen «Todesarten»-Fragmente. ZurGeschichte des reflexiven und zyklischen Erzählens bei Ingeborg Bachmann. In: Ingeborg Bachmanns «Malina». Hrsg. von Andrea Stoll. Frankfurt/M. 1992, S. 188-209, hier: S. 199-202.

6. - Vgl. Verf.: Die Strukturgenese des «Malina» - Romans. Zur Entstehungsgeschichte von Ingeborg Bachmanns «Todesarten» - «Ouvertüre». In: Ingeborg Bachmann- Neue Beiträge zu ihrem 
Werk. Internationales Symposion Münster 1991. Hrsg. vomVerf. und Hubert Ohl. Würzburg 1993, S. 147-165, hier: S. $149 f$.

7. - Ingeborg Bachmann: Besichtigung einer alten Stadt. In: Text + Kritik, H. 6 (2. Aufl. 1971), S. 18-20.

8. - Vgl. Verf.: Die Strukturgenese des «Malina» - Romans (Anm. 6), S. 164f.

9. - Helmut Heißenbüttel: Über Ingeborg Bachmanns Roman «Malina». In: Text + Kritik, H.6 (2. Aufl. 1971), S. 25-27, hier: S. 27.

10. - Vgl. Andrea Stoll: Erinnerung als ästhetische Kategorie des Widerstandes imWerk Ingeborg Bachmanns. Frankfurt/M. und Bern 1991, S. 23 und 251.

11. - Vgl. Gespräche und Interviews (Anm. 1), S. 66, und Verf.: «Die Schwarzkunst der Worte» Zur Barbey- und Rimbaud-Rezeption in Ingeborg Bachmanns «Todesarten» - Zyklus. In: Jahrbuch der Grillparzer-Ges., 3. Folge, 17 (1989), S. 127-162.

12. - Walter Grünzweig: Die vergebliche Enttrümmerung beschädigter Kinderköpfe: Nationalsozialismus in den Werken Gert Hofmanns. In: German Studies Review, 12 (1989), S. 55-67, hier: S. 65.

13. - In dem 'exotischen Zeitroman' «Vor der Regenzeit» (1988) und dem deutlicher autobiographischen Roman «Der Kinoerzähler» (1990) werden diese beiden Linien des Werks weitergeführt.

14. - Aus Gert Hofmanns Werken wird unter Verwendung folgender Siglen zitiert: $\mathrm{D}=$ Die Denunziation. Reinbek 1982; UV = Unsere Vergeßlichkeit. Darmstadt und Neuwied 1987. Vgl. zur Textstruktur sowie zu dem (zeit-) geschichtlichen und intertextuellen Anspielungshorizont detailliert: Frank Vogel: Geschichtsdarstellung und Zeitkritik im Erzählwerk Gert Hofmanns. Magisterarbeit Münster 1989.

15. - Gert Hofmann: Die Überflutung. 4 Hörspiele. Frankfurt/M. 1981, S. 165-214; Fuhlrotts Vergeßlichkeit. Portrait eines uns bekannten Kopfes. Erzählungen. Graz 1981; Unsere Vergeßlichkeit, in: Merkur, 35 (1981), S. 389-396. Die Verlagerung des Schauplatzes von dem Berlin dieser älteren Texte in das «F.» des Romans dürfte mit der politischen Debatte über die Zerstörung des ehemals jüdischen Frankfurter Westends 1985 zusammenhängen (vgl. K. Briegleb: «Aufbruch 1968» [Anm. 2], S. 124f.).

16. - Die Sigle JM steht für die Ausgabe München: dtv 1987.

17. - Vgl. Sigrid Berka: Mythos-Theorie und Allegorik bei Botho Strauß. Wien 1991, passim; Walter Rügen: Die Vermessung des Innenraums. Zur Prosa von Botho Strauß. Würzburg 1991, S. 178-181; Claus Sommerhage: Odeon oder der verschollene Krug. Über Botho Strauß' romantische Poetik der Erinnerung. In: Sinn und Form, 43 (1991), S. 177-196.

18. - Vgl. S. Berka: Mythos-Theorie und Allegorik (Anm. 17), S. 19.

\section{RÉSUMÉS}

Identitätsproblematik und Zeitkritik werden in Ingeborg Bachmanns «Todesarten» -Projekt der späten 60er Jahre sowie in Gert Hofmanns zeit- und geschichtskritischem Erzählwerk und Botho Strauß' Roman «Der junge Mann» der 80er Jahre durch unterschiedliche und doch durch ihre Selbstreflexivität und durch den allen drei Autoren gemeinsamen exemplarischen Bezug auf den Nationalsozialismus vergleichbare Ausfaltungen einer Dialektik der Erinnerung vermittelt, die über traditionelle Formen des Gesellschafts- und Zeitromans hinausführt und komplexe 
ästhetische Reflexionsräume der Verschränkung von Geschichtlichkeit und Subjektivität hervorbringt. Die Auseinandersetzung mit der «Geschichte im Ich» und der Erfahrung psychischer und sozialer Gewalt in der Nachkriegsgesellschaft in Ingeborg Bachmanns Roman «Malina» findet in Gert Hofmanns Erzählung «Die Denunziation» und in seinem Roman «Unsere Vergeßlichkeit» eine eigenständige Fortschreibung in einer Geschichtsund Bewußtseinskritik, die als eine Dialektik gegenzsätzlicher und zugleich komplementärer Formen des Erinnerns und 'Vergessens' gesellschaftlicher Ausgrenzungs-, Verdrängungs- und Vernichtungsstrategien entfaltet wird. Demgegenüber hebt Botho Strauß seine Zeit- und Gesellschaftskritik in einer grundlegenden Zeitbegriffs- und Gleichzeitigkeits-Reflexion auf, deren 'postmoderne' Struktur wechselseitiger Spiegelungen einander entgegengesetzter und zugleich aufeinander bezogener Modelle des Umgangs mit Zeit und Geschichte dennoch auf das Verhältnis von Lebens- und Zeitgeschichte zurückbezogen bleibt, das die Erinnerungspoetiken Bachmanns und Hofmanns problematisieren.

Dans le projet des «formes de mort » d'I. Bachmann à la fin des années soixante, dans l'œuvre narrative "d'actualité » de Gert Hofmann, ainsi que dans le roman Der junge Mann de Botho Strauss des années quatre-vingt, la problématique de l'identité et la critique du temps trouvent leur expression dans les différentes formes d'une dialectique du souvenir différente pour chacun et pourtant comparable dans la réflexion qu'ils mènent et dans l'attitude exemplaire que ces trois auteurs ont eue à l'égard du national-socialisme. Cette dialectique, dépassant les formes traditionnelles du roman social et du roman d'actualité, renvoie à des réflexions esthétiques complexes sur l'imbrication de l'historicité et de la subjectivité. Le débat sur l'histoire inscrite dans le Moi et sur l'expérience de la violence psychique et sociale dans la société d'après-guerre, tel qu'il apparaît dans le roman d'Ingeborg Bachmann Malina, trouve dans le récrit de Gert Hofmann Die Denunziation et dans son roman Unsere Vergeßlichkeit un prolongement spécifique à travers la critique de la conscience de l'histoire; cette critique se développe comme une dialectique de formes contradictoires et en même temps complémentaires du souvenir et de l'«oubli» des stratégies d'exclusion, de refoulement et d'anéantissement de la société. A l'opposé, Botho Strauß transforme sa critique du temps et de la société en une réflexion fondamentale sur la notion de Temps et des temps parallèles, dont la structure "postmoderne ", qui fait que les modèles de relation au temps et à l'histoire à la fois opposés et interdépendants se reflètent mutuellement, continue de se référer au rapport entre Histoire générale et histoire individuelle qui problématisent les poétiques du souvenir de Bachmann et Hofmann.

\section{AUTEUR}

\section{DIRK GÖTTSCHE}

Westphälische-Wilhelms-Universität Münster 